\title{
The Application of IHA in Grid Cloud Computing Task Decomposition and Scheduling Based on Bionics
}

\author{
Kailun Shi \\ North China Electric Power University, Beijing, China \\ Email: 1099246657@qq.com
}

Received April 2015

\begin{abstract}
Based on the large amount and variations of the power grid task as well as its requirement of realtime performance and economic benefit, we make a further improvement and expansion of IHA (Improved Heuristic Algorithm) on the combination of bionics in genetic engineering and evolution to solve the decomposing and scheduling problems. Firstly, we transform those complex decomposing problems into the operational optimal solution problem by IHA to decrease the rate of running into the local optimal solution [1]. In task scheduling, we classify the sub-tasks by the emergency degree for resource allocation, which not only largely reduces the calculation and resource cost but also improves working efficiency and the speed of execution [2]. Finally, we select optimal scheduling scheme by the Fitness function defined about time and cost.
\end{abstract}

\section{Keywords}

Grid Task, Cloud Computing, IHA, Bionics, Cloudsim

\section{Introduction}

With the development of modern science and technology, biological sciences and power system began to appear increasingly close relationship. Like a large biological system, power system is carrying out complicated but orderly work every moment, which distributes numerous power grid computing tasks in a wide range of resource pool constituted of many computers. Then corresponding solutions and scheduling mechanism plays in a coordinating role to make the users obtain corresponding cloud resources on demand. In the same time, tasks should be given the real-time and effective solution. Genetic engineering and evolution mechanism are combined with cloud computing to solve the decomposing and scheduling problems [3]. The close connection between modern biological science and power system is elaborated by simulation and results analysis.

\section{Background}

At present, the characteristics of our power grid is large regional difference of distribution and complex relationship among each part. Based on powerful storage and computing capacity of intelligent cloud, we can distribute the sub-tasks of each node to many distributed computers to greatly increase the utilization rate of re- 
sources, therefore, improves the economic benefit of power grid. However, the existing grid computing task decomposition algorithm easily run into the problem of local optimal solution, which affects the normal management of resource request from users shows a performance of power grid. The task scheduling under the cloud computing is collecting all kinds of information from power grid and giving a feedback to the power cloud. The computers will calculate the relevant parameters and indicators based on the information stored in cloud resources, and give valid instructions by feasible judgment of operation state both in safety and economy to achieve a relatively stable state by necessary adjustments.

Inappropriate task scheduling strategy can lead to waste of cloud resources and even influence the quality of power grid, which may seriously affected the enterprise's economic benefit.

\section{The Basic Principle of IHA}

The basic principle is to partition computing tasks. Namely, sub-tasks are stored in one node of the and/or tree, and the user request task decomposition means choosing the right point of division and building structure of the tree. We estimate overall consumption after using heuristic method to find a division of the task, when dividing point selection is improper, sub-task will not be able to perform and be regard as infinity of consumption. Repeat these steps until all the sub-tasks couldn't be further subdivided, then we think the task decomposition is completed.

Task decomposition has two important steps: first, in order to ensure the optimal solution is for overall tasks, every step must meet the constraint conditions of relevant task decomposition; Second, in order to improve the efficiency of resource requesting and distributing and minimize the costs, we should carry on reasonable classification and allocation to these divided tasks.

\section{The Combination of Bionics}

Considering some related concept of genetic engineering in biological science, gene cutting, we need use a socalled "scalpel" to do molecule specificity cutting for some specific nucleotide sequences on the DNA, whose scientific name is restriction endonuclease. We regard the initial power grid computing tasks as a DNA analogy, on which there are one or some nodes of operation, monitoring, protection, distribution, marketing, etc. The constraint to divide the task can be regard as "scalpel" to do specific cutting for the computing task which conforms to the corresponding node cutting constraints. Then we take one of these nodes to do further division with the IHA and look for task dividing point of a node. If the node has at least one task segmentation point and the sub-tasks can be retrieved in the cloud after segmentation, then this point can be determined as the division point of tasks. Do further division until all sub-task can't be divided any more, task decomposition process is over. If the divided sub-task can't be retrieved in the cloud, rework following process above until all its tasks can be retrieved in the cloud. Finally, polish sub-tasks tree according to the consumption to perfect the decomposition process.

Based on the natural selection in biological science, we expand and perfect the original algorithm to make it has a good performance both in reasonable decomposition but also for optimal resource allocation. We define these alternative task scheduling schemes as a species. First of all, we encode the initialized population, then we define a fitness function to evaluate whether the individual of population is competitive enough. The task scheduling scheme with highest fitness function value above all alternative schemes will give the most appropriate scheduling command.

\section{Flow Chart of the Algorithm}

Certain grid tasks and resources must correspond to different solutions. Because the degree of emergency and importance is directly related to the real-time requirements of tasks, thus affecting the priority level of a task in the process of solving [4]. We first make a division of emergency degree for the sub-tasks which can't be divided any more, then a resource allocation scheme is presented. Next, to avoid the full arrangement for each task as well as make a selection from the alternative schemes created by the full arrangement, therefore saving a lot of time and computing resources to improve the efficiency and saving resources. Finally, we take further screening of these relatively reasonable scheduling scheme by using the theory of natural selection and define the fitness function considering time and cost to give a standard to screen out optimal solution as well as give scheduling command [5]. The process is shown in Figure 1. 


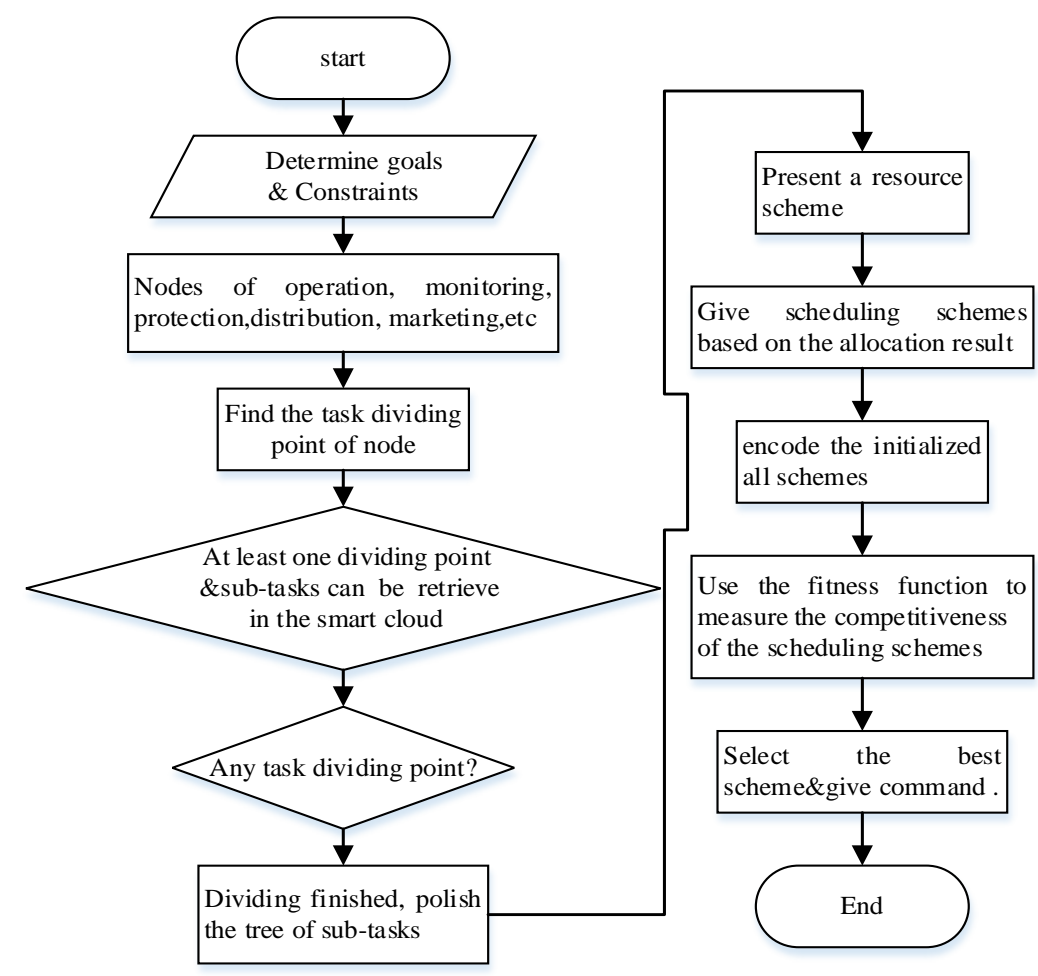

Figure 1. Flow chart of the algorithm.

\section{Summary}

This paper is based on grid computing task decomposing and scheduling problems, and gives a combination of bionics to perfect IHA. In the scheduling process, We classify the sub-tasks by the emergency degree for resource allocation to reduce the resource consuming and improve the work efficiency. Then, we define the fitness function of the time and cost to evaluate competitiveness of a individual scheduling scheme and select the most optimal one. Finally, this algorithm is verified by simulation experiments in the process of grid task computing can give a reasonable decomposition. In terms of resource allocation, we present a new method. In a word, the task decomposition and reasonable allocation of resources in grid cloud environment can improve the stability and efficiency of the power system operation, and improve the economic benefit of power enterprise, which laid a good foundation for the application of intelligent cloud in power grid.

\section{References}

[1] Su, X.H. and Zhang, H.L. (2012) Improvement of Cloud Computing Task Decomposition Algorithm. Electronic Design Engineering, 23, 47-49.

[2] Li, J., Li, P.-W., Li, Y.-K., Yu, J.-G. and Shao, Z.X. (2012) Cloud Computing in the Application of the Smart Grid Research. Mathematics in Practice and Theory, 13, 123-129.

[3] Zhu, Y.H. (2013) Task Scheduling Research of Differential Evolution Algorithm and Its Application in Cloud Computing. Lanzhou Jiaotong University.

[4] Yu, H.F. (2011) Scheduling Algorithm Research of Cloud Computing Based on Effect Function. Science and Technology Information, 3, 35+47.

[5] Kang, Y.M. and Hu, J. (1997) Task Decomposition, Task Scheduling and Parallel Algorithm Design. Computer Development \& Applications, 3, 42-44. 